\title{
A Clinical Test for Diagnosis of Humeroradial Joint Lesions in the Presence of Lateral Elbow Pain: Single-Case Design with Arthroscopic Confirmation
}

\author{
Holly Jonely ${ }^{*}$, Jean-Michel Brismée ${ }^{2}$, David Lutton ${ }^{3}$ \\ ${ }^{1}$ Department of Health, Human Function \& Rehabilitation Sciences, The George Washington School of Medicine \& Health \\ Sciences, Washington, DC, USA \\ ${ }^{2}$ Center for Rehabilitation Research, Texas Tech University Health Sciences Center, Lubbock, TX, USA \\ ${ }^{3}$ Department of Orthopaedic Surgery, The George Washington School of Medicine \& Health Sciences, Washington, DC, USA \\ Email: *hjonely@gwu.edu
}

How to cite this paper: Jonely, H., Brismée, J.-M. and Lutton, D. (2018) A Clinical Test for Diagnosis of Humeroradial Joint Lesions in the Presence of Lateral Elbow Pain: Single-Case Design with Arthroscopic Confirmation. International Journal of Clinical Medicine, 9, 162-174.

https://doi.org/10.4236/ijcm.2018.93014

Received: January 29, 2018

Accepted: March 9, 2018

Published: March 12, 2018

Copyright $\odot 2018$ by authors and Scientific Research Publishing Inc. This work is licensed under the Creative Commons Attribution International License (CC BY 4.0).

http://creativecommons.org/licenses/by/4.0/

\begin{abstract}
INTRODUCTION: Lateral epicondylosis is a common cause of lateral elbow pain, however, some patients fail conservative management. One reason, many pathologies have similar presentations. There are no valid and reliable clinical tests to differentiate between tendopathy and arthropathy. This single case design looks at the utilization of the humeroradial joint (HRJ) distraction test to diagnose HRJ chondropathy. CASE SUMMARY: 38-year-old male recreational athlete with persistent lateral elbow pain, impaired motion, crepitus and locking. Disability of the Arm Shoulder Hand Questionnaire (DASH): 13.33/100. DASH Sport Module, 68.75/100. Differential Diagnosis: Chondropathy of the HRJ with loose body, based on history of elbow pain and locking, non-capsular pattern of limitation of motion and a positive HRJ distraction test. Intervention: loose body manipulation improved pain free range of motion with continued remarkable HRJ distraction test; referral to orthopedic surgeon. Plain radiographs and CT demonstrated a loose body and marked cartilage thinning within the HRJ. Arthroscopic removal of the loose body and plica resection was performed. Surgical exploration confirmed Grade 3 cartilage lesions within the radial head without involvement of the extensor muscle group. Post-operative physical therapy focused on restoration of accessory joint motion and education on return to functional and recreational activity. OUTCOMES: Full range and normal joint accessory motion, DASH score and DASH Sport Module score reduced to 8.33 and 25, 12-weeks post-operative. CONCLUSION: Arthroscopic exploratory surgery is recom-
\end{abstract}


mended for chronic lateral elbow pain resistant to conservative management; however, patients who present with pain in the absence of a positive clinical examination and radiographic findings benefit least from arthroscopic evaluation. No clinical test(s) have been validated in the literature to diagnose HRJ lesions. This report is the first to propose the use of a new clinical test for HRJ lesion, with confirmation using imaging and arthroscopy. Level 4 Case Report.

\section{Keywords}

Epicondylalgia, Chondropathy, Loose Body, Plica

\section{Introduction}

Lateral epicondylosis is one of the most common causes of lateral elbow pain associated with diminished grip strength, painful gripping, painful resisted wrist extension, radial deviation, extension of the middle finger and pain with palpation over the lateral epicondyle at the origin of the extensor carpi radialis brevis (ECRB) [1]-[6]. Treatment begins with bracing, icing, non-steroidal anti-inflammatory medications, corticosteroid injections, activity modification, resistance training, ergonomic evaluation and physical therapy [3] [7]. It is well established that lateral epicondylosis may take 3 - 12 months to resolve, leading to frustration for both clinicians and patients. The delay in resolution of symptoms may simply be the natural course of the disease; however, it also could be related to an inaccurate diagnosis. There are several pathologies that afflict the lateral elbow including: posterior interosseous nerve entrapment, posterolateral rotatory instability, plica syndrome, Panner's disease, radiocapitellar joint chondromalacia, degeneration, osteochondral fracture, loose body, osteochondritis dissecans or cervical dysfunctions [8] [9] [10]. It is therefore important for clinicians to have knowledge of the anatomy of the elbow complex and the pathophysiology of associated conditions in order to make an accurate diagnosis and propose appropriate plans of care.

Cadaveric, arthroscopic and imaging studies suggest that lateral elbow pain can present with and without involvement of the extensor muscle group. Goodfellow and Bullough [11] first described post mortem evidence of articular cartilage degeneration within the elbow complex. They reported that observed degeneration appeared to be age dependent and primarily reserved to the humeroradial joint (HRJ) [11]. Various authors [8] [12] [13] have used arthroscopy to evaluate patients who presented clinically with chronic lateral elbow pain resistant to conservative care. Rajeev et al observed that $59 \%$ of patients who reported additional symptoms of locking (25\%), crepitus and stiffness (45\%) also exhibited articular cartilage degeneration, with $88 \%$ of those patients exhibiting degenerative changes exclusively within the HRJ [13]. Degenerative changes within the HRJ were found within the radial head in $68 \%$ of patients and within the ca- 
pitulum in $69 \%$ of patients. Observed degeneration varied from Grade 1 - 3 and included partial and full thickness tears. Only $36 \%$ of patients exhibited extensor origin inflammation. Other pathological findings included radial head plica (16\%) [13], synovitis (55\% - 84\%) [8] [12] [13], loose body (7.1\% - 16\%) [8] [12] [13], adhesions (4\%) [13], valgus extension overload (5\%) [8] [12] and osteophytes (12\% - 14\%) [8] [12] [13].

The clinician begins to develop a working diagnosis based on the patient's history and clinical examination prior to including more expensive and/or invasive diagnostic procedures. However, presently there is not a valid and reliable clinical test, which can be utilized to differentiate between lateral elbow pain secondary to extensor tendopathy and/or arthropathy. Plain radiographs often fail to assist in excluding involvement of unicompartmental lateral degeneration [13] in patients with chronic lateral elbow pain. The following case study demonstrates how including the HRJ distraction and the HRJ plica tests within the clinical examination of a patient with chronic lateral elbow pain allowed for early diagnosis and management.

\section{Case Report}

A 38-year-old left hand dominant male $(185 \mathrm{~cm}, 99.79 \mathrm{~kg})$ competitive and recreational athlete (weight lifting, long distance cycling and marathon runner) presented to physical therapy via direct access with complaints of left lateral elbow pain. He reported a bicycle accident 7 -months prior where he rolled across the hood of a vehicle striking his left elbow on the hood and then landing on the pavement on his feet. Initially he reported acute elbow pain, without impaired function which resolved within a few weeks. However, more recently he began complaining of recurrent pain, loss of elbow range of motion (ROM), crepitus and intermittent painful locking when performing certain workout activities. Locking was associated with sharp pain and inability to fully extend the elbow. The patient relieved symptoms of locking with active ROM of the wrist and forearm. Following an incidence of locking, the pain occurred with simple grasping pad to pad as well as movement of the elbow. Pain remained elevated for a few weeks limiting his ability to participate in any upper body weight lifting. The pain was described as sharp in nature over the lateral aspect of the elbow with worst/least intensity ratings of 7 out of 10 and 2 out of 10, respectively, using the numeric pain rating scale. Disability was measured using the Disability of the Arm Shoulder Hand Questionnaire (DASH) [14]. The score was $13.33 \%$ out of $100 \%$, where $100 \%$ indicates total disability. The DASH Sport Module was $68.75 \%$. The patients' past medical history was otherwise unremarkable.

\subsection{Examination}

After obtaining informed consent, the patient underwent a physical therapy examination. The clinical examination included an unremarkable cervical screen which was used to rule out referred pain secondary to a history of trauma and 
the persistent nature of symptoms. This was followed by an assessment of active and passive range of motion of the elbow and forearm. Range of motion data will provide the examiner with information on the quality, quantity and provocation of motion and willingness to move. Pain was observed when testing elbow flexion and extension with forearm pronation and supination as well during testing of active and passive forearm pronation and supination. It was hypothesized that since symptom provocation occurred with the addition of forearm pronation and supination, pathology of the HRJ was suspect. Resistance testing to the wrist extensors was then performed to assess for impairment of strength or provocation of symptoms. These tests were remarkable for pain. Since the patient was observed to have pain with both testing targeting the HRJ as well as wrist extensor muscle group, the examiner would need a tissue differentiation test to differentiate between a primary HRJ pathology versus a musculotendinous pathology. Therefore, the HRJ distraction test (Figure 1 \& Supplemental Video (https://youtu.be/ObVXC9yKTIg) and HRJ plica compression test (Figure 2 \& Supplemental Video) were performed. Remarkable examination findings are listed in Table 1.

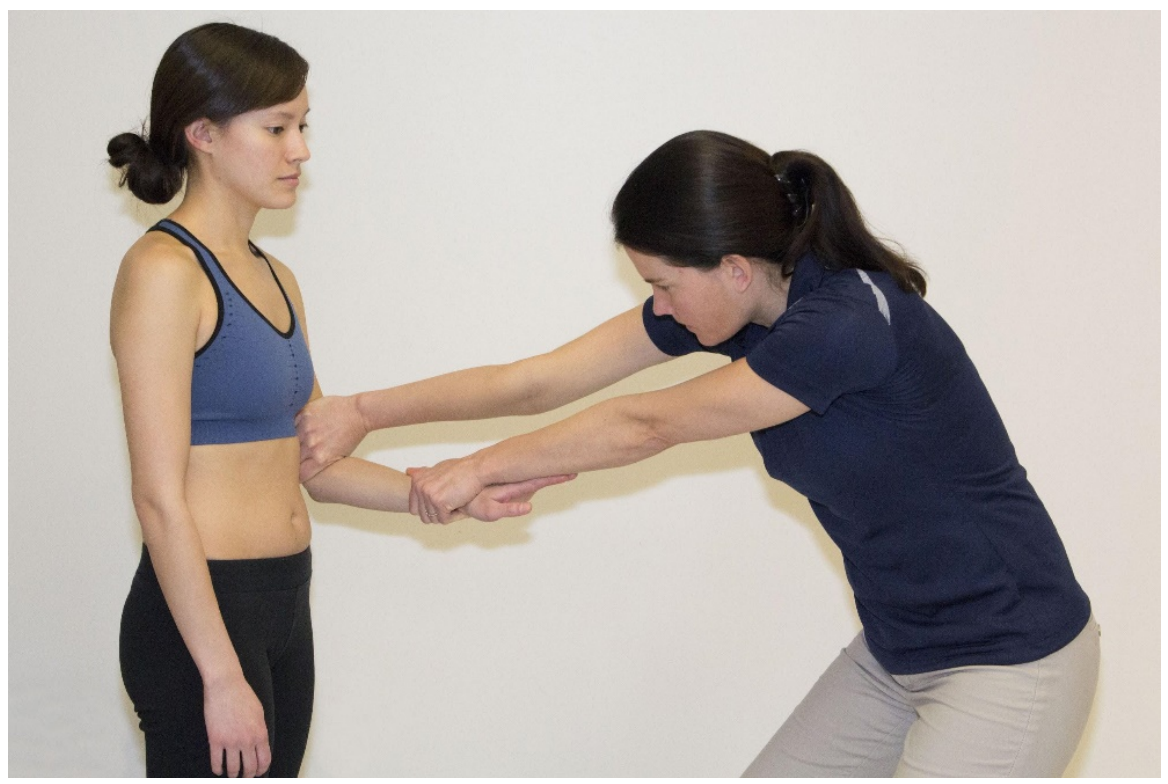

Figure 1. Humeroradial joint distraction test. In standing, the clinician grasps the distal end of the radius using a lumbrical grip and allows the dorsum of the patient's hand to rest under the clinician's forearm while stabilizing the distal humerus with the opposite hand. The patient is then instructed to extend their wrist into the clinician's forearm gradually applying as much resistance as possible. The clinician monitors the amount of force generated. The patient is quarried whether pain was reproduced and asked to rate the intensity on a scale from $0-10$. The patient is then asked to cease the contraction. The clinician then applies a traction force to the radius distally in line with the radius. While maintaining the traction force the patient is asked again to extend their wrist into the clinicians' forearm gradually applying as much resistance as possible. The clinician monitors the amount of force generated. The patient is quarried as to whether the test reproduces their symptoms and asked to rate the intensity on a scale from $0-10$. 


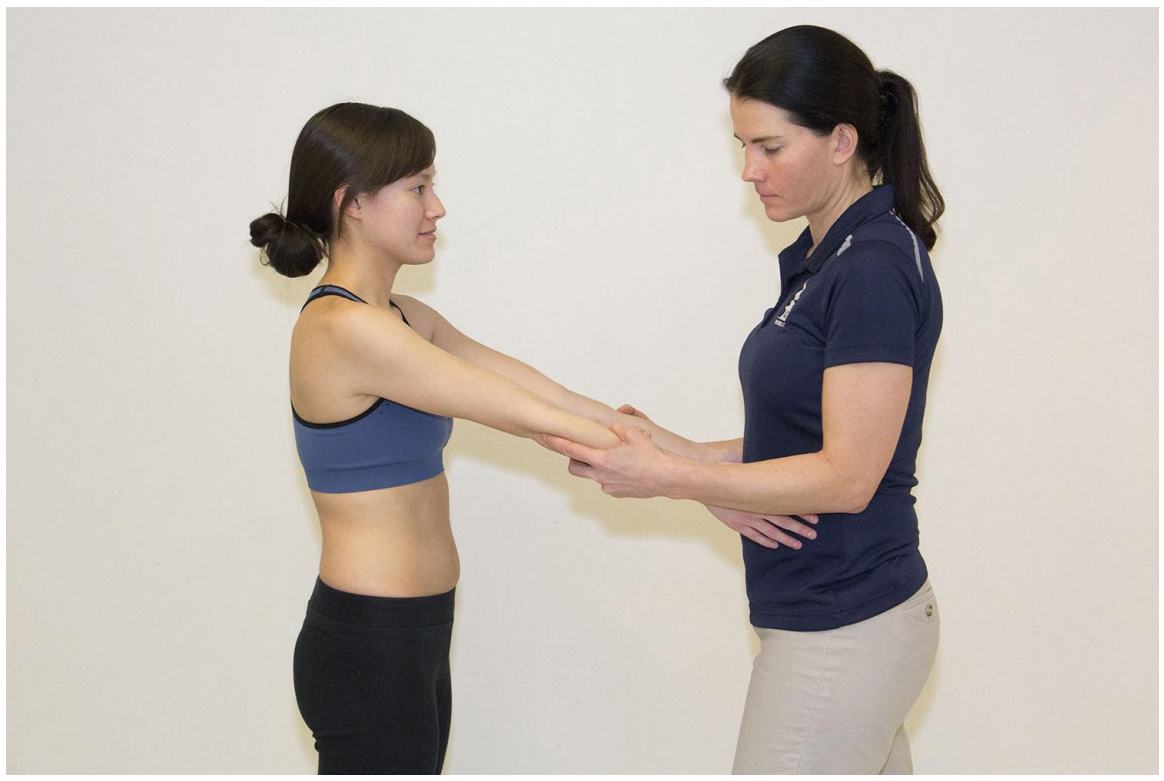

Figure 2. Humeroradial joint plica test. The clinician faces the patients and supports the proximal forearm of each arm in the palm of their hand. The patient's elbows are flexed to 90 degrees and their hands rest on the waist of the clinician. The clinician palpates the humeral radial joint line laterally using the index finger. The clinician then passively moves the patient's elbows from 90 degrees flexion to full extension while maintaining compression of the index fingers over the lateral humeroradial joint line. At the end range of extension, the clinician assesses for any reproduction of pain as well as whether the palpating digit is able to remain within the indentation of the humeroradial joint line.

Table 1. Clinical examination. Only remarkable findings are reported (pain $=+$ minimum, ++ moderate, and +++ severe).

\begin{tabular}{cc}
\hline Tests & Outcomes \\
Passive Elbow Flexion & $130^{\circ}$, end feel firm \\
With Pronated Forearm & Pain ++ \\
With Supinated Forearm & Pain ++ \\
Passive Elbow Extension & $10^{\circ}$, end feel firm \\
With Pronated Forearm & Pain ++ \\
Passive Forearm Pronation at 80 Degrees Elbow Flexion & Pain ++ \\
Passive Forearm Supination at 80 Degrees Elbow Flexion & $80^{\circ}$, Pain + \\
Resistive Wrist Extension & $80^{\circ}$, Pain + \\
Resistive Wrist Radial Deviation & Pain + \\
Resistive Wrist Ulnar Deviation & Pain + \\
HRJ Distraction Test (Figure 1 \& Video $)$ & Pain +
\end{tabular}

\subsection{Clinical Impression \#1}

Following the clinical examination the assessment included the diagnosis of 
chondropathy of the HRJ vs loose body secondary to a traumatic event. The assessment was based on the remarkable HRJ compression test suggesting a possible painful hypertrophic plica. However, the history of painful locking did not exclude the diagnosis of a loose body and therefore would need further exploration. The etiology of a loose body can have many origins [15]: idiopathic, Panner's disease, osteochondrosis dessicans, synovial osteochondromatosis, flake fracture and villonodular synovitis. Proposed treatments include an intra articular injection, loose body manipulation, joint specific treatments to restore limits in mobility and surgical excision [15] [16]. The proposed diagnosis of a loose body was based on a history of pain in the elbow and mechanical symptoms including locking and catching, a non-capsular pattern of limitation of motion [17] and the presence of a positive HRJ distraction test. The clinician then opted to perform a loose body manipulation of the HRJ (Supplemental Video). Following the manipulation technique, the client exhibited increased pain free active and passive ROM of the elbow and forearm. The intervention was successful, but did not resolve issues completely. Secondary to the patient's history of trauma and the continued consideration of a possible HRJ arthropathy, the patient was referred to an orthopedic surgeon for further evaluation which included diagnostic imaging.

\subsection{Clinical Impression \#2}

Plain radiographs demonstrated the presence of a loose body along the HRJ and medial humeral ulnar joint (Figure 3). Osteophytes were also observed along the tip of the coronoid and olecranon. Further evaluation by CT scan showed a 1.2 $\mathrm{cm}$ curvilinear ossification in the medial joint space of the HRJ as well as marked cartilage thinning (Figure 4). The addition of diagnostic imaging confirmed diagnosis of a loose body and chondropathy of the HRJ.

\subsection{Intervention}

Left elbow arthroscopy was performed and included removal of a loose body from the radiocapitellar joint (Figure 5), HRJ plica resection, synovial resection over the anterior HRJ and osteophyte resection over the coronoid and olecranon tip (Figure 6). Surgical exploration confirmed the patient exhibited Grade 3 cartilage lesions within the radial head (Figure 7) without involvement of the extensor muscle group. Physical therapy was initiated 2 weeks following surgery. The patient received six sessions of physical therapy over a four-week period, which included joint mobilization to address end range limitations of the HRJ and graded return to exercise. The patient denied any adverse effects from treatment.

\subsection{Outcome}

At discharge, his DASH and DASH Sport Module score were reduced to $8.33 \%$ and 25\%, respectively. Both questionnaires reached MDC and MCID at one-year 


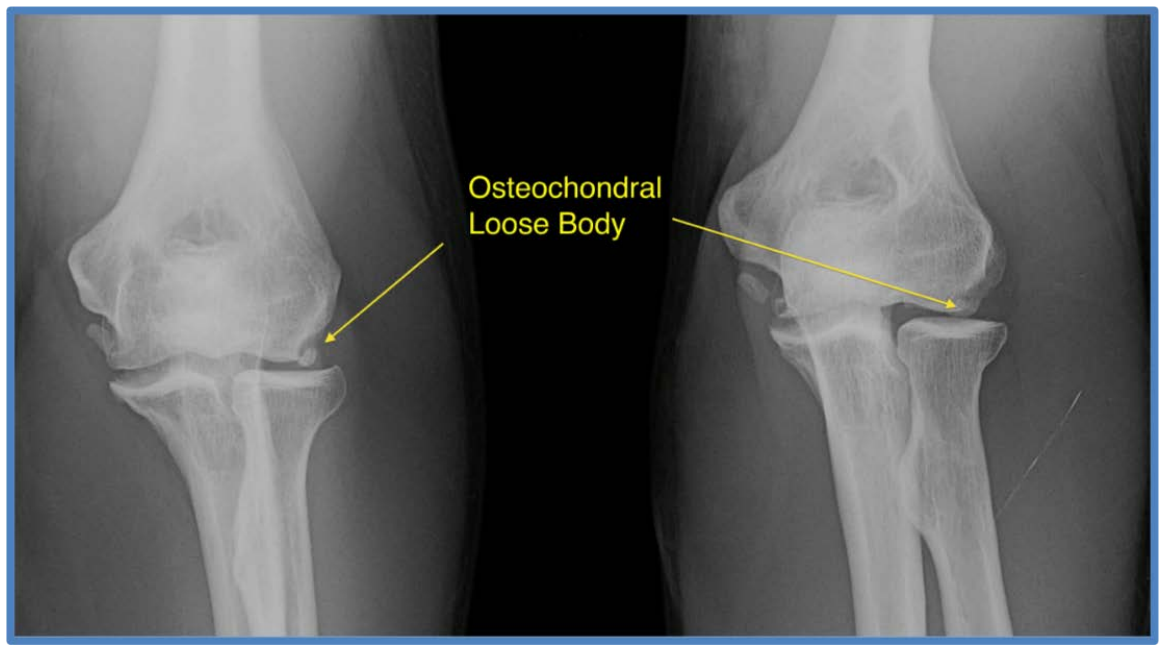

Figure 3. HRJ radiograph with observed osteochondral loose body, denoted by the object at the end of the arrowhead(s).

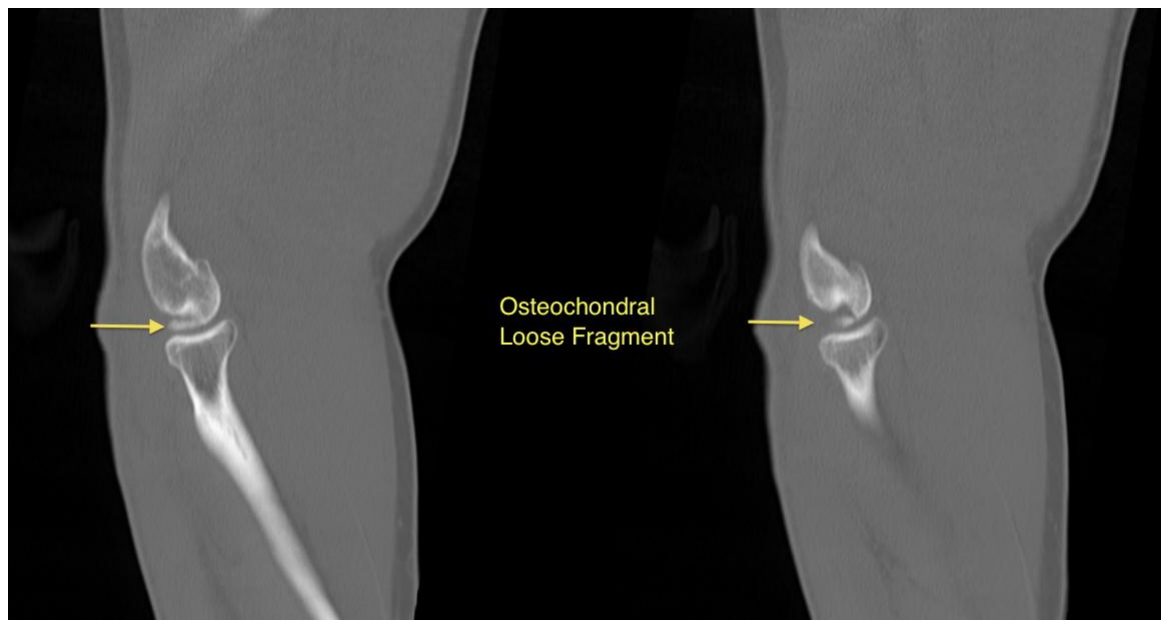

Figure 4. CT scan with observed osteochondral loose body, denoted by the object at the end of the arrowhead(s).

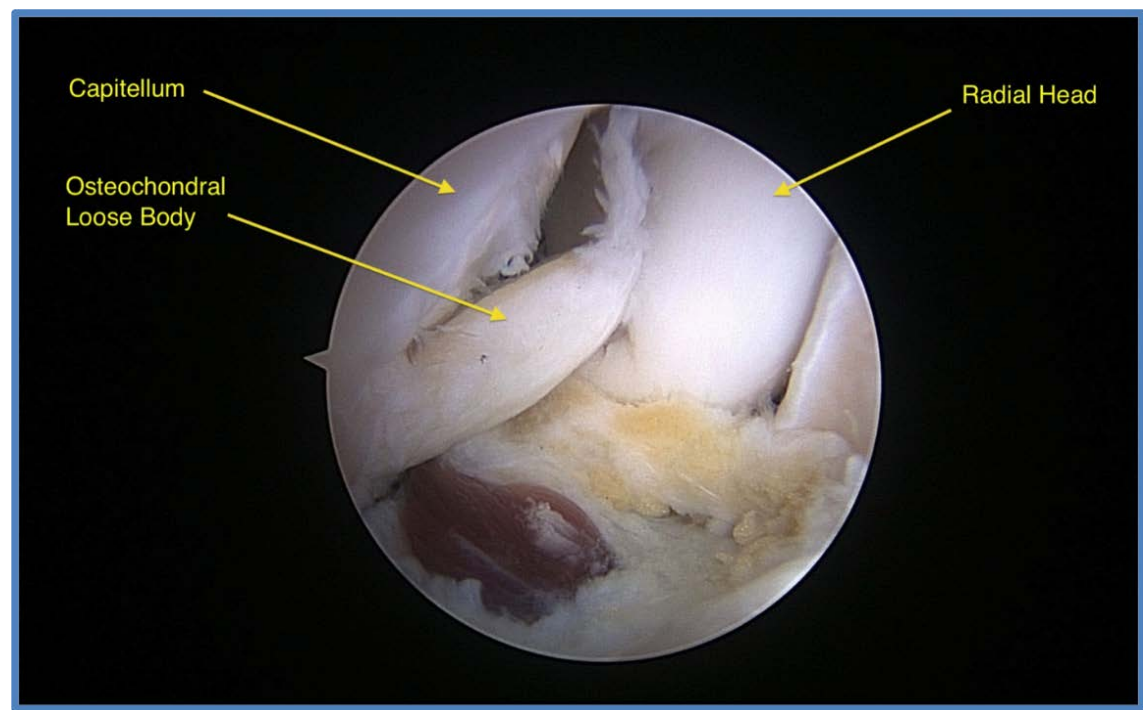

Figure 5. Arthroscopic image with observed osteochondral loose body. The structure at the end of each arrowhead corresponds to the designated label. 


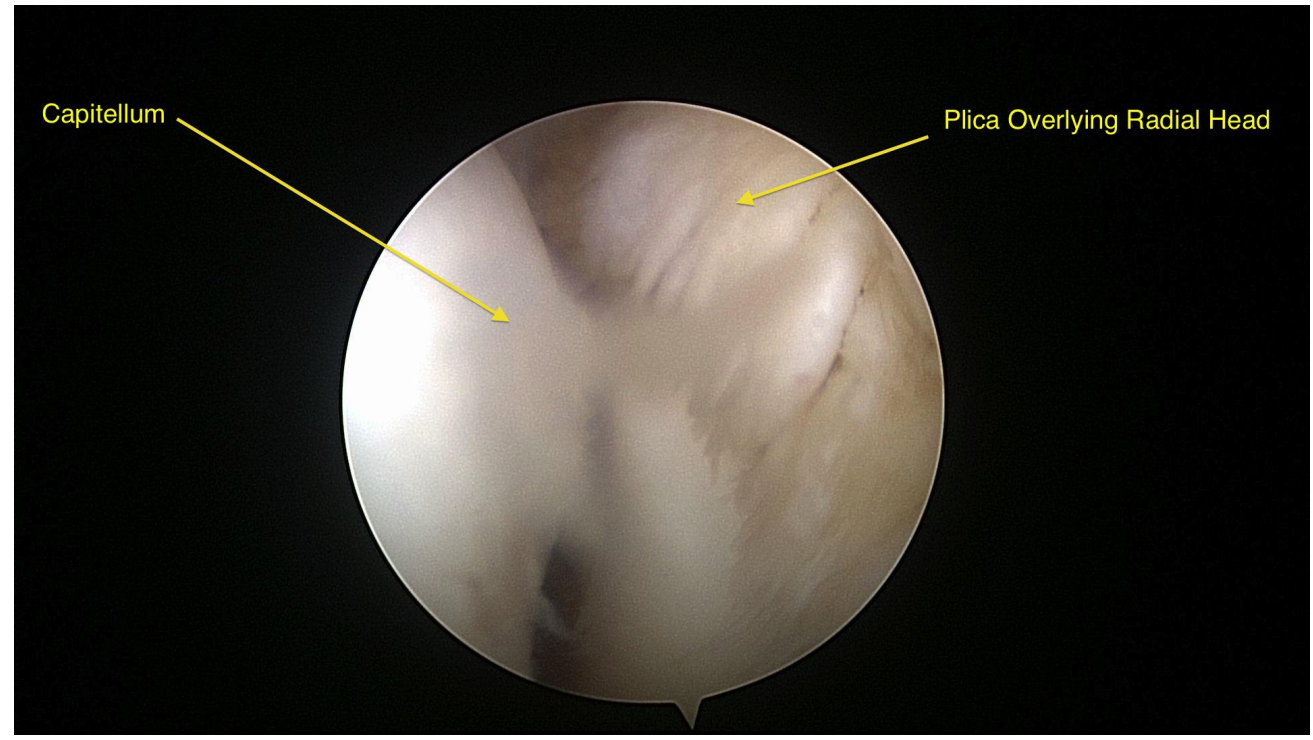

Figure 6. Arthroscopic image with observed hypertrophic plica. The structure at the end of each arrowhead corresponds to the designated label.

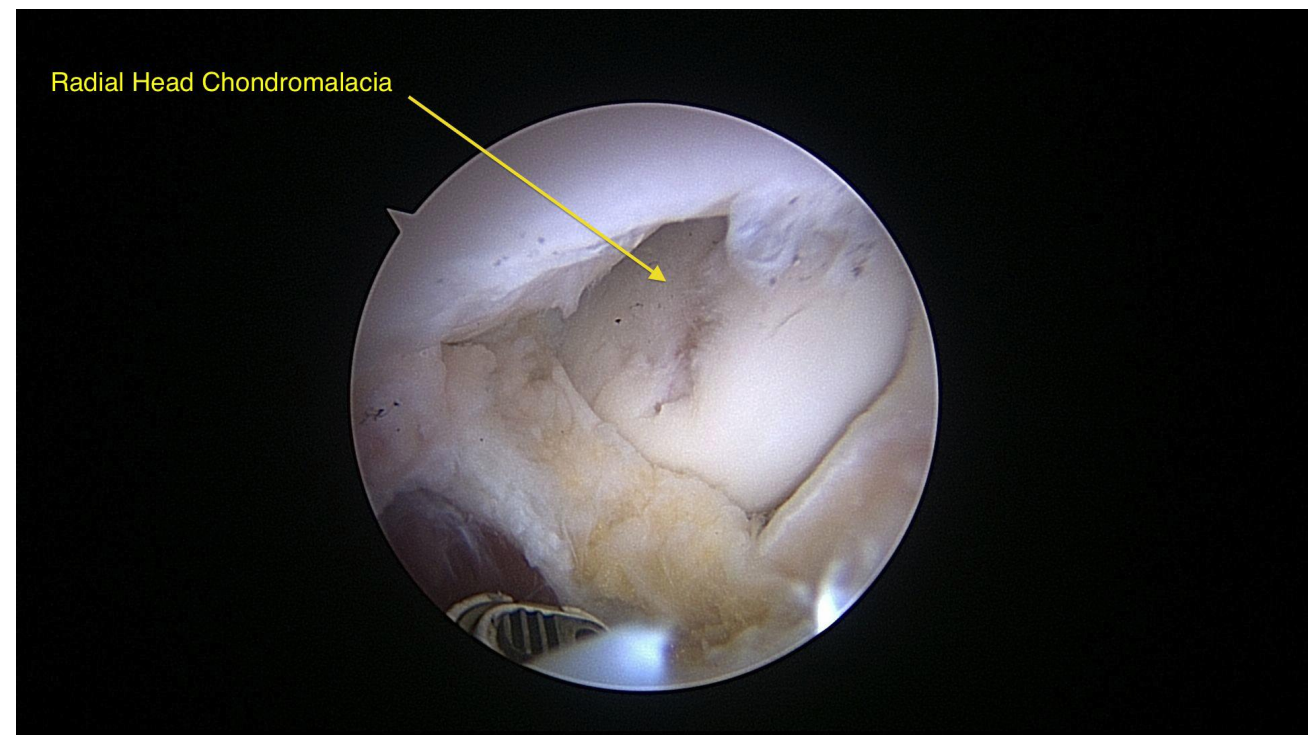

Figure 7. Arthroscopic image with observed Grade 3 radial head cartilage lesion, denoted by the area of tissue observed at the end of the arrowhead.

follow-up representing a change of 13.33 and 68.75 points [14]. There were no limitations in mobility observed upon reassessment at 12 weeks post op, the lack of observed ROM in the elbow was secondary to soft tissue restrictions from biceps brachii hypertrophy. Table 2 lists outcomes associated with the case monitored over the course of one year.

\section{Discussion and Literature Review}

Arthroscopic exploratory surgery is often recommended for chronic lateral elbow pain resistant to conservative management; however, patients who present with pain in the absence of a positive clinical examination and radiographic 
Table 2. Patient care outcomes.

\begin{tabular}{cccccc}
\hline Test \& Measure & Pre-Operatively & $\begin{array}{c}\mathbf{1} \text { week } \\
\text { postop }\end{array}$ & $\begin{array}{c}\mathbf{6} \text { weeks } \\
\text { postop }\end{array}$ & $\begin{array}{c}12 \text { weeks } \\
\text { postop }\end{array}$ & $\mathbf{1}$ year \\
\hline $\begin{array}{c}\text { Elbow PROM } \\
\text { Extension-Flexion }\end{array}$ & $10-130$ & $\begin{array}{c}30-120 \\
\text { empty end feel firm end feel soft end feel soft end feel }\end{array}$ \\
$\begin{array}{c}\text { Elbow PROM } \\
\text { Pronation-Supination }\end{array}$ & $80-80$ & $70-80$ & Full & Full & Full \\
HRJ Distraction Test & Positive & Not Tested & Negative & Negative & Negative \\
HRJ Plica Compression Test & Positive & Not Tested & Negative & Negative & Negative \\
DASH & 13.33 & Not Tested & 8.33 & 1.66 & 0 \\
DASH Sport Module & 68.75 & Not Tested & 25 & 6.25 & 0 \\
\hline
\end{tabular}

findings benefit least from arthroscopic evaluation [18]. This single case report is the first to propose the use of new clinical tests to assist in differentiating between etiologies of lateral elbow pain. The tests included the HRJ distraction test and HRJ joint plica compression test. Clinical findings were then confirmed with imaging and arthroscopy.

Plain radiograph, CT and MR arthrography have shown little success in assisting in diagnosis of Grade 1 or 2 chondral changes [13] [18] [19]. However, Waldt et al found that the use of CT and MR arthrography to be equally sensitive and specific (87/94\% and 85/95\%, respectively) in detecting Grade 3 and 4 lesions [19]. Rajeev et al also concluded that the presence of a normal plain radiograph was not relevant in excluding the diagnosis of arthropathy [13] because radiographs failed to detect early signs of joint arthrosis. Additionally, the use of plain radiograph, CT and MR arthrography were found to lack reliability and accuracy in diagnosing the presence of loose bodies. When compared to arthroscopic evaluation, radiography was often unable to distinguish between an osteophyte and loose body and unable to detect whether the particle was intra or extra articular [18] [19]. These authors also concluded that CT and/or MR arthrography were not any more effective than plain radiograph in diagnosing a loose body (overall sensitivity $88 \%-100 \%$ and specificity $20 \%-70 \%$ ). Therefore, to avoid unnecessary surgical and expensive diagnostic procedures in the absence of a definitive clinical examination, an accurate clinical diagnosis through the use of valid and reliable tissue differentiation tests is suggested. In this patient case the clinician hypothesized the presence of a HRJ pathology. This was based on the patient history of trauma, loss of range of motion and painful locking in the elbow. The application of the HRJ plica compression and HRJ distraction test supported the clinicians reasoning, directed initial care including a loose body manipulation of the elbow and later need for referral to an orthopedist to confirm diagnosis when effects of the initial intervention plateaued. Diagnostic imaging confirmed the suspicion of HRJ pathology as well as loose body.

A few studies examined the relationship between the diagnosis of lateral epi- 
condylosis and the presence of radiocapitellar joint degeneration [11] [13] [20] [21] [22] [23]. Quintart et al [24] found during surgical examination of 17 patients that $65 \%$ of the patients exhibited extension limits of the elbow and $53 \%$ of patients displayed radial head chondropathy [24]. More recently Sasaki et al performed a retrospective evaluation of 31 patients who had undergone arthroscopic surgery secondary to recalcitrant lateral epicondylosis. All patients' diagnoses were based on a history of lateral elbow pain, pain with resisted wrist extension and palpation over the lateral epicondyle. All subjects exhibited increased MRI signal intensity of the ECRB at its origin, but did not exhibit evidence of HRJ or humeroulnar joint arthropathy. Evidence of cartilage injury was observed later during arthroscopic evaluation; capitellum (65\%) and radial head (81\%). Capitellum degeneration has also been independently associated with the absence of ECRB tear in persons with lateral epicondylosis [23].

Resistance testing and soft tissue length assessments are common clinical tests performed during a musculoskeletal examination to assess tolerance of the musculotendinous components to stress and strain. When examining the wrist extensor group in patients with lateral elbow pain, the authors propose pain during resistance testing or gripping, could be secondary to either dysfunction of the musculotendinous unit or increased compressive forces within the HRJ. To assist in tissue differentiation, the HRJ distraction test was proposed by authors. The test assesses the change in pain during resisted wrist extension with and without HRJ distraction. It is hypothesized that the addition of a traction force applied to the radius would have no effect on pain of musculotendinous origin but would decrease or eliminate pain of osteocartilagenous origin secondary to decreased compressive load within the joint. In this patient case elbow arthroscopy confirmed the absence of extensor muscle group involvement and presence of Grade 3 chondral changes of the humeral head. Therefore, the addition of the HRJ distraction test in this patient case, assisted the clinician in differentiating between impairments of the wrist extensor group from HRJ arthropathy.

Many researchers have proposed that the plica [24] and synovial fold [25]-[30] may contribute to lateral elbow pain. Quintart et al [24] observed during surgical exploration that $76 \%$ of patients diagnosed with lateral epicondylitis exhibited evidence of a plica within the HRJ. Duparc et al [25] found the presence of a synovial fold within the HRJ in $86 \%$ of the 50 cadaveric specimens examined. When Duparc et al [25] compared cadaveric specimens to five live subjects who had recently undergone surgery for epicondylalgia they found that the resected synovial fold exhibited signs of inflammation, increased thickness and numerous nerve fibers as compared to the cadaveric specimens. Clarke [28] found in his surgical exploration of patients diagnosed with a loose body that it was actually the presence of a fibrotic synovial fringe impinged within the HRJ that caused catching, locking and pain rather than a loose body [28].

Since synovial plica syndrome presentation is similar to lateral epicondylosis, careful evaluation for proper diagnosis is warranted. Some authors have palpated 
a snapping plica around 80 - 110 degrees of elbow flexion as the elbow is moved from supination and extension to pronation and flexion in $25 \%-50 \%$ of patients [29] [30] [31]. Many tests and measures can cause irritation of the synovial fold or plica at the HRJ; therefore, the authors suggest the inclusion of HRJ plica test to assist with differentiation. The test purports to compress the plica and or synovial fold within the HRJ space during passive elbow extension, producing lateral elbow pain. Also, in the presence of a hypertrophic plica or posterior capsule, the clinician's palpating finger will be pushed out of the indentation created by the HRJ line when the patient's elbow is fully extended. When symptomatic, a plica or synovial fold may be treated with an injection, iontophoresis with dexamethasone, loose body manipulation and/or surgical resection [24] [25] [29] [30]. In this case, the patient responded well to a loose body manipulation; it is therefore plausible that the plica became impinged within the HRJ during active or passive extension motions. The presence of a HRJ plica was observed and resected during arthroscopic exploration.

\section{Conclusion}

Although this case review is limited by a single case design, it is the first report providing confirmation of the clinical findings of a HRJ distraction and compressive test using imaging and arthroscopic exploration in a patient case where a HRJ lesion and hypertropic plica were suspected. Prospective studies are needed to determine the predictive validity of the HRJ distraction test and HRJ plica test to assist in differentiating between lateral elbow pain secondary to extensor tendopathy or radiocapitellar joint arthropathy.

\section{Disclosure}

The authors declare that there is no conflict of interest regarding the publication of this article. Informed consent was obtained from all individual participants included in the study.

\section{References}

[1] Calfee, R., Patel, A., DaSilva, M. and Akelman, E. (2008) Management of Lateral Epicondylitis: Current Concepts. Journal of the American Academy of Orthopaedic Surgeons, 16, 19-29. https://doi.org/10.5435/00124635-200801000-00004

[2] Levin, D., Nazarian, L.N., Miller, T.T., O’Lane, P.L., Feld, R.I., Parker, L. and McShane, J.M. (2005) Lateral Epicondylitis of the Elbow: US Findings. Radiology, 237, 230-234. https://doi.org/10.1148/radiol.2371040784

[3] Raman, J., MacDermid, J.C. and Grewal, R. (2012) Effectiveness of Different Methods of Resistance Exercises in Lateral Epicondylosis-A Systematic Review. Journal of Hand Therapy, 25, 5-25. https://doi.org/10.1016/j.jht.2011.09.001

[4] Ahmad, Z., Siddiqui, N., Malik, S., Abdus-Samee, M., Tytherleigh-Strong, G. and Rushton, N. (2013) Lateral Epicondylitis. A Review of Pathology and Management. The Bone \& Joint Journal, 95, 1158. https://doi.org/10.1302/0301-620X.95B9.29285

[5] Tosti, R., Jennings, J. and Sewards, J. (2013) Lateral Epicondylitis of the Elbow. American Journal of Medicine, 126, 357. 
https://doi.org/10.1016/j.amjmed.2012.09.018

[6] Dorf, E., Chhabra, A., Golish, S., McGinty, J. and Pannunzio, M. (2007) Effect of Elbow Position on Grip Strength in the Evaluation of Lateral Epicondylitis. The Journal of Hand Surgery, 32, 882. https://doi.org/10.1016/j.jhsa.2007.04.010

[7] Snyder, K.R. and Evans, T.A. (2012) Effectiveness of Corticosteroids in the Treatment of Lateral Epicondylosis. Journal of Sport Rehabilitation, 21, 83-88. https://doi.org/10.1123/jsr.21.1.83

[8] Kaminsky, S.B. and Baker Jr., C.L. (2003) Lateral Epicondylitis of the Elbow. Techniques in Hand \& Upper Extremity Surgery, 7, 179-189.

https://doi.org/10.1097/00130911-200312000-00009

[9] Van Hofwegen, C., Baker III, C.L. and Baker Jr., C.L. (2010) Epicondylitis in the Athlete's Elbow. Clinics in Sports Medicine, 29, 577-597. https://doi.org/10.1016/j.csm.2010.06.009

[10] Hausman, M. and Lang, P. (2014) Examination of the Elbow: Current Concepts. The Journal of Hand Surgery, 39, 2534. https://doi.org/10.1016/j.jhsa.2014.04.028

[11] Goodfellow, J. and Bullough, P. (1967) The Pattern of Ageing of the Articular Cartilage of the Elbow Joint. The Bone \& Joint Journal, 49, 175-181.

[12] Baker, C.J. and Baker III, C.L. (2008) Long-Term Follow-Up of Arthroscopic Treatment of Lateral Epicondylitis. The American Journal of Sports Medicine, 36, 254-260. https://doi.org/10.1177/0363546507311599

[13] Rajeev, A. and Pooley, J. (2009) Lateral Compartment Cartilage Changes and Lateral Elbow Pain. Acta Orthopædica Belgica, 75, 37-40.

[14] Beaton, D., Davis, A., Hudak, P. and McConnell, S. (2001) The DASH (Disability of the Arm, Shoulder, and Hand) Outcome Measure: What Do We Know about It Now? British Journal of Hand Therapy, 6, 109-118. https://doi.org/10.1177/175899830100600401

[15] Leissing, C., Savioz, D. and Fritschy, D. (1997) Arthroscopic Removal of Intra-Articular Loose Foreign Bodies of the Elbow. Revue De Chirurgie Orthopedique Et Reparatrice De L'Appareil Moteur, 83, 707-711.

[16] Greis, P.E., Halbrecht, J. and Plancher, K.D. (1995) Arthroscopic Removal of Loose Bodies of the Elbow. Orthopedic Clinics of North America, 26, 679-689.

[17] Cyriax, J.H. (1993) Cyriax's Illustrated Manual of Orthopaedic Medicine. Butterworth \& Heinemann, Oxford.

[18] Dubberley, J., Faber, K., Patterson, S., Garvin, G., Bennett, J., Romano, W., MacDermid, J.C. and King, G.J.W. (2005) The Detection of Loose Bodies in the Elbow: The Value of MRI and CT Arthrography. The Journal of Bone and Joint Surgery. British Volume, 87, 684-686. https://doi.org/10.1302/0301-620X.87B5.14851

[19] Waldt, S., Bruegel, M., Ganter, K., Kuhn, V., Link, T.M., Rummeny, E.J. and Woertler, K. (2005) Comparison of Multislice CT Arthrography and MR Arthrography for the Detection of Articular Cartilage Lesions of the Elbow. European Radiology, 15, 784-791. https://doi.org/10.1007/s00330-004-2585-9

[20] Newman, J.H. and Goodfellow, J.W. (1975) Fibrillation of Head of Radius as One Cause of Tennis Elbow. British Medical Journal, 2, 328-330. https://doi.org/10.1136/bmj.2.5966.328

[21] Grewal, R., MacDermid, J.C., Shah, P. and King, G.J. (2009) Functional Outcome of Arthroscopic Extensor Carpi Radialis Brevis Tendon Release in Chronic Lateral Epicondylitis. Journal of Hand Surgery (American Volume), 34, 849-857.

https://doi.org/10.1016/j.jhsa.2009.02.006 
[22] Lattermann, C., Romeo, A.A., Anbari, A., Meininger, A.K., McCarty, L.P., Cole, B.J. and Cohen, M.S. (2010) Arthroscopic Debridement of the Extensor Carpi Radialis Brevis for Recalcitrant Lateral Epicondylitis. Journal of Shoulder and Elbow Surgery, 19, 651-656. https://doi.org/10.1016/j.jse.2010.02.008

[23] Sasaki, K., Onda, K., Ohki, G., Sonoda, T., Yamashita, T. and Wada, T. (2012) Radiocapitellar Cartilage Injuries Associated with Tennis Elbow Syndrome. Journal of Hand Surgery (American Volume), 37, 748-754.

https://doi.org/10.1016/j.jhsa.2012.01.005

[24] Quintart, C., Reignier, M. and Baillon, J.M. (1998) Tennis Elbow: Surgical Findings in 17 Cases and Etiopathogenetic Hypothesis. Acta Orthopædica Belgica, 64, 170-174.

[25] Duparc, F., Putz, R., Michot, C., Muller, J. and Freger, P. (2002) The Synovial Fold of the Humeroradial Joint: Anatomical and Histological Features, and Clinical Relevance in Lateral Epicondylalgia of the Elbow. Surgical and Radiologic Anatomy, 24, 302-307. https://doi.org/10.1007/s00276-002-0055-0

[26] Isogai, S., Murakami, G., Wada, T. and Ishii, S. (2001) Which Morphologies of Synovial Folds Result from Degeneration and/or Aging of the Radiohumeral Joint: An Anatomic Study with Cadavers and Embryos. Journal of Shoulder and Elbow Surgery, 10, 169-181. https://doi.org/10.1067/mse.2001.112956

[27] Akagi, M. and Nakamura, T. (1998) Snapping Elbow Caused by the Synovial Fold in the Radiohumeral Joint. Journal of Shoulder and Elbow Surgery, 7, 427-429. https://doi.org/10.1016/S1058-2746(98)90037-4

[28] Clarke, R. (1988) Symptomatic, Lateral Synovial Fringe (Plica) of the Elbow Joint. Arthroscopy, 4, 112-116. https://doi.org/10.1016/S0749-8063(88)80077-X

[29] Antuna, S. and O'Driscoll, S. (2001) Snapping Plicae Associated with Radiocapitellar Chondromalacia. Arthroscopy, 17, 491-495. https://doi.org/10.1053/jars.2001.20096

[30] Steinert, A., Goebel, S., Rucker, A. and Barthel, T. (2010) Snapping Elbow Caused by Hypertrophic Synovial Plica in the Radiohumeral Joint: A Report of Three Cases and Review of Literature. Archives of Orthopaedic and Trauma Surgery, 130, 347-351. https://doi.org/10.1007/s00402-008-0798-0

[31] Cerezal, L., Rodriguez-Sammartino, M., Canga, A., Capiel, C., Arnaiz, J., Cruz, A. and Rolon, A. (2013) Elbow Synovial Fold Syndrome. American Journal of Roentgenology, 201, W88-W96. https://doi.org/10.2214/AJR.12.8768

\section{Supplemental Video}

Video. Instructional video for the performance of the "Humeroradial Joint Distraction Test", "Humeroradial Joint Plica Test" and "Loose Body Manipulation of the Elbow". This information will allow the reader to more accurately apply the psychomotor skill associated with the performance of these tests and intervention (https://youtu.be/ObVXC9yKTIg). 